\title{
Energy principle of ferroelectric ceramics and single domain mechanical model
}

\author{
Feng Liu · Haijun Li · T. C. Wang
}

Received: 30 December 2006 / Accepted: 5 April 2007 / Published online: 24 July 2007

(C) Springer-Verlag 2007

\begin{abstract}
Many physical experiments have shown that the domain switching in a ferroelectric material is a complicated evolution process of the domain wall with the variation of stress and electric field. According to this mechanism, the volume fraction of the domain switching is introduced in the constitutive law of ferroelectric ceramic and used to study the nonlinear constitutive behavior of ferroelectric body in this paper. The principle of stationary total energy is put forward in which the basic unknown quantities are the displacement $u_{i}$, electric displacement $D_{i}$ and volume fraction $\rho_{I}$ of the domain switching for the variant $I$. Mechanical field equation and a new domain switching criterion are obtained from the principle of stationary total energy. The domain switching criterion proposed in this paper is an expansion and development of the energy criterion. On the basis of the domain switching criterion, a set of linear algebraic equations for the volume fraction $\rho_{I}$ of domain switching is obtained, in which the coefficients of the linear algebraic equations only contain the unknown strain and electric fields. Then a single domain mechanical model is proposed in this paper. The poled ferroelectric specimen is considered as a transversely isotropic single domain. By using the partial experimental results, the hardening relation between the driving force of domain switching and the volume fraction of domain switching can be calibrated. Then the electromechanical response can be calculated on the basis of the calibrated hardening relation. The results involve the electric butterfly shaped curves of axial strain versus axial electric field, the hysteresis loops
\end{abstract}

The project supported by the National Natural Science Foundation of China (10572138).

F. Liu · H. Li · T. C. Wang $(\varangle)$

LNM, Institute of Mechanics, Chinese Academy of Sciences,

Beijing 100080, China

e-mail: tcwang@imech.ac.cn of electric displacement versus electric filed and the evolution process of the domain switching in the ferroelectric specimens under uniaxial coupled stress and electric field loading. The present theoretic prediction agrees reasonably with the experimental results given by Lynch.

Keywords Ferroelectric ceramic · Domain switching . The volume fraction of domain switching - Single domain mechanical model

\section{Introduction}

Ferroelectric ceramics are used in many electric components such as sensors, actuators and transducers owing to their coupled electromechanical character. And as the core device of smart structures they are used in aerospace, precise instruments, automatic control and micro electromechanical system and so on. When the applied electric field closes to or exceeds the coercive electric field, the classical linear piezoelectricity theory is not suitable. There have been extensive research efforts in the field of constitutive modeling and numerical simulation of the behavior of ferroelectric ceramics.

Many theories were proposed to explain the nonlinear behavior of the ferroelectric ceramics. The different types of constitutive models for the ferroelectric ceramics can be classified into two groups. One group is on the basis of the micro-electro-mechanical behavior of grains and the second on the macro phenomenological model.

Early a micro-electro-mechanical model was proposed by Hwang et al. [1]. They established the energy criterion of the domain switching using the energy difference before and 
after the domain switching,

$E_{k} \Delta D_{k}^{s}+\sigma_{i j} \Delta \gamma_{i j}^{s} \geq 2 E_{c} P_{s}$,

where $\Delta \gamma_{i j}^{s}$ and $\Delta D_{k}^{s}$ are the changes of spontaneous strain and spontaneous electric displacement before and after the domain switching, respectively, $E_{c}$ is the coercive electric field, $P_{S}$ is the spontaneous polarization. Lu et al. [2] developed Hwang's [1] switching criterion. They distinguished the energy barriers of $90^{\circ}$ and $180^{\circ}$ domain switching. The results given by them matched well with those under uniaxial mechanical loading. Chen et al. [3] introduced the volume fraction of various kinds of domains as internal variables describing the pattern of the internal rearrangement resulting from the domain switching and studied the nonlinear behavior of polycrystalline ferroelectrics. Huo and Jiang [4] considered that every crystal had different kinds of domains and that the mass fractions of the various domains were the internal variables, so the average polarization of each crystal could be expressed as the linear function of the mass fraction and the domain switching corresponds to changes of the volume fraction of domains. Huber et al. [5] developed the ferroelectric constitutive model on the basis of the domain wall motion that is similar to the crystal slip. They thought that there were several variants in a tetragonal crystal and the volume fraction of each variant was $c_{I}$. They used a selfconsistent analysis to estimate the macroscopic response of tetragonal crystals under a variety of loading paths. Huber and Fleck [6,7] improved this model. The micro-electromechanical models described some aspects of the behavior of ferroelectric ceramics [8-11]. Micro-electro-mechanical model reflects the physical essence of the nonlinear behavior of the ferroelectric material. But in order to make the calculation accuracy, those methods require thousands of crystallite grains. Hence the finite element simulations cost too much computation time and are not efficient.

On the other hand, instead of micro-electro-mechanical based constitutive model the phenomenological models with fewer internal variables are developed. Chen and his collaborators [12-14] proposed the phenomenological model to describe the coupled electromechanical characters of ferroelectric materials. They used the remanent polarization and remanent strain as the internal variables and they only considered the uniaxial loading. Another attempt to study the nonlinear constitutive behavior of the ferroelectric materials was made by Bassiouny and Maugin [15,16]. They presented a thermodynamical phenomenological theory to describe the constitutive behavior of the ferroelectric ceramics. The theory considers both the plastic and electrichysteresis effects in the form of "plasticity". A simple macroscopic constitutive model with remanent polarization and remanent strain as internal variables was developed by Kamlah and his coworkers [17-19]. The model can be used to study the fully coupled electromechanical boundary value problem. A phenomenological theory was also presented by Cocks and McMeeking [20]. The model is established in the framework of traditional incremental plasticity. Landis [21] introduced nine internal variables corresponding to the components of remanent strain and remanent polarization to match all of the types of behaviors of ferroelectric ceramics in uniaxial loading. A simplified formulation with fewer internal variables is derived by McMeeking and Landis [22]. In the model only three internal variables are employed by linking the remanent strain to the remanent polarization. Due to this simplicity the switching criterion can be expressed by a modified electric field variable and the kinematic hardening potential can be given as a function of the remanent polarization. A detailed review on recent advances in the nonlinear constitutive modeling of ferroelectric material was given by Landis [23]. So far all the phenomenological theories predicted well for the uniaxial mechanical loading and the uniaxial electric loading. But there are some deviations between the theoretic prediction and experimental results under uniaxial combined electromechanical loading.

\section{Constitutive law and energy principle}

\subsection{The volume fraction of domain switching}

The physical experiments given by Miller and Weinreich [24] and Hayashi [25] showed that the domain switching in the ferroelectric ceramics was a complicated process. It was composed by four stages: nucleation, longitudinal growth, transverse expansion and merging of new domains [26]. Hence the domain switching in each single domain is an evolution process of the domain wall motion.

Following Hwang et al. [8], Chen and Lynch [10], Kamlah [18] and McMeeking and Landis [22], the ferroelectric ceramic is assumed to be isotropic elastic and isotropic dielectric for simplicity. But the piezoelectric coefficient $d_{k i j}$ will be changed due to the domain switching. Firstly consider the domain switch only in one direction. Suppose the material element is a single domain zone before switching. The spontaneous polarization, the spontaneous strain and piezoelectricity coefficient are $D_{i}^{s(0)}, \gamma_{i j}^{s(0)}$ and $d_{i j k}^{(0)}$, respectively. The corresponding quantities will be $D_{i}^{s(I)}, \gamma_{i j}^{s(I)}$ and $d_{i j k}^{(I)}$ after whole domain in the material element has switched. If the volume fraction of the switched domain in the material element is $\rho_{I}$, the spontaneous polarization, spontaneous strain and piezoelectric coefficient of the material element will be

$$
\begin{aligned}
D_{i}^{s} & =\left(1-\rho_{I}\right) D_{i}^{s(0)}+\rho_{I} D_{i}^{s(I)}=D_{i}^{s(0)}+\rho_{I} \Delta D_{i}^{s(I)}, \\
\gamma_{i j}^{s} & =\left(1-\rho_{I}\right) \gamma_{i j}^{s(0)}+\rho_{I} \gamma_{i j}^{s(I)}=\gamma_{i j}^{s(0)}+\rho_{I} \Delta \gamma_{i j}^{s(I)}, \\
d_{i k l} & =\left(1-\rho_{I}\right) d_{i k l}^{(0)}+\rho_{I} d_{i k l}^{(I)}=d_{i k l}^{(0)}+\rho_{I} \Delta d_{i k l}^{(I)},
\end{aligned}
$$


where symbol $\Delta$ is the change of related physical quantity before switching and after whole domain of the material element having switched. Note that the material element considered here includes thousands of crystalline grains. So the volume fraction of domain switching may not directely depend upon that of the grain boundaries, the orientations of crystal lattices and the twinning mode. If the material element is a grain, the reader is referred to the contribution by Zhang [27].

In fact, the domain may switch both in the $90^{\circ}$ and $180^{\circ}$ directions. And the domain may switch in several potential orientations during $90^{\circ}$ switching. Suppose the volume fraction of $180^{\circ}$ switching is $\rho_{1}$. If there are $N-190^{\circ}$ switching, the volume fraction of $90^{\circ}$ switching can be expressed as $\rho_{I}(I \in 2,3, \ldots, N)$.

Similar to Eq. (2), the spontaneous electric displacement, the spontaneous strain and the piezoelectric coefficient of the material element can be written as follows

$$
\begin{aligned}
& D_{i}^{s}=D_{i}^{s(0)}+\sum_{I=1}^{N} \rho_{I} \Delta D_{i}^{s(I)}, \\
& \gamma_{i j}^{s}=\gamma_{i j}^{s(0)}+\sum_{I=1}^{N} \rho_{I} \Delta \gamma_{i j}^{s(I)}, \\
& d_{i k l}=d_{i k l}^{(0)}+\sum_{I=1}^{N} \rho_{I} \Delta d_{i k l}^{(I)},
\end{aligned}
$$

where $I=1$ corresponds to $180^{\circ}$ switching, $I \in 2,3, \ldots, N$ are corresponding $90^{\circ}$ switching in the $I$ th orientation.

The constitutive law of the ferroelectric ceramic can be described as following

$$
\begin{gathered}
\gamma_{i j}-\gamma_{i j}^{s(0)}-\sum_{I=1}^{N} \rho_{I} \Delta \gamma_{i j}^{s(I)}=S_{i j k l} \sigma_{k l}+d_{k i j} E_{k} \\
=S_{i j k l} \sigma_{k l}+\left(d_{k i j}^{(0)}+\sum_{I=1}^{N} \rho_{I} \Delta d_{k i j}^{(I)}\right) E_{k}, \\
D_{i}-D_{i}^{s(0)}-\sum_{I=1}^{N} \rho_{I} \Delta D_{i}^{s(I)}=d_{i k l} \sigma_{k l}+\varepsilon_{i k} E_{k} \\
=\left(d_{i k l}^{(0)}+\sum_{I=1}^{N} \rho_{I} \Delta d_{i k l}^{(I)}\right) \sigma_{k l}+\varepsilon_{i k} E_{k},
\end{gathered}
$$

where $S_{i j k l}$ and $\varepsilon_{i k}$ are the elastic compliance and dielectric permittivity tensor measured at constant electric field and constant stress, respectively. Tensor $d_{i k l}$ is the piezoelectric coefficient at constant stress. In general the displacement $u_{i}$ and electric potential $\phi$ are used as the basic unknown quantities in finite element method. Hence the second type constitutive law is needed in which the basic unknown quantities are the strain $\gamma_{i j}$ and electric field $E_{i}$. We have

$$
\begin{aligned}
\sigma_{i j}= & C_{i j m n}\left(\gamma_{m n}-\gamma_{m n}^{s}\right)-C_{i j m n} d_{k m n} E_{k}, \\
D_{i}= & D_{i}^{s}+C_{m n k l} d_{i k l}\left(\gamma_{m n}-\gamma_{m n}^{s}\right) \\
& +\left(\varepsilon_{i k}-d_{k m n} e_{i m n}\right) E_{k},
\end{aligned}
$$

where $C_{i j m n}$ is the elastic modulus tensor measured at constant electric field, $e_{i m n}$ is the piezoelectric coefficient tensor at constant strain $e_{i m n}=C_{m n k l} d_{i k l}$.

\subsection{Principle of stationary total energy}

Suppose the volume and boundary of the ferroelectric body are $V$ and $S$, respectively. When no body forces and free charges are present, the field equations can be written as

$\sigma_{i j, j}=0$,

$D_{i, i}=0$.

The mechanical boundary conditions are:

$\sigma_{i j} n_{j}=\bar{t}_{i} \quad$ on $S_{\sigma}$,

$u_{i}=\bar{u}_{i}$ on $S_{u}$,

where $\sigma_{i j}$ is the components of stress, $\bar{t}_{i}$ is the prescribed traction on $S_{\sigma}, \bar{u}_{i}$ is the prescribed displacement on $S_{u}$.

The electrical boundary conditions are:

$$
\begin{aligned}
& D_{i} n_{i}=-\bar{\omega} \text { on } S_{\omega}, \\
& \phi=\bar{\phi} \text { on } S_{\phi},
\end{aligned}
$$

where $D_{i}$ is the electric displacement, $\bar{\omega}$ is the prescribed surface free charge on $S_{\omega}, n_{i}$ is the unit normal to the surface $S, \bar{\phi}$ is the prescribed electric potential on $S_{\phi}$.

The internal energy density can be expressed as following [28]

$$
\begin{aligned}
U=\frac{1}{2}[ & \sigma_{i j}\left(\gamma_{i j}-\gamma_{i j}^{s(0)}-\sum_{I=1}^{N} \rho_{I} \Delta \gamma_{i j}^{s(I)}\right) \\
& \left.+E_{k}\left(D_{k}-D_{k}^{s(0)}-\sum_{I=1}^{N} \rho_{I} \Delta D_{k}^{s(I)}\right)\right] .
\end{aligned}
$$

When the domain switching is considered, the total energy is

$$
\begin{aligned}
\Phi= & \int_{V} U \mathrm{~d} V-\int_{S_{\sigma}} \bar{t}_{i} u_{i} \mathrm{~d} S+\int_{S_{\phi}} \bar{\phi} D_{i} n_{i} \mathrm{~d} S \\
& +\int_{V}\left(\sum_{I=1}^{N} \rho_{I} W_{\mathrm{cr}}^{(I)}\right) \mathrm{d} V
\end{aligned}
$$

where $W_{\mathrm{cr}}^{(1)}$ is the critical energy barrier that the single domain of per unit volume must overcome when the polarization of the whole domain has switched by $180^{\circ}, W_{\mathrm{cr}}^{(I)}(I=2,3, \ldots$, $N$ ) is the critical energy barrier that the single domain of per unit volume must overcome when the whole domain has switched by $90^{\circ}$ in the $I$ th variant orientation. The last term is the additional energy due to domain switching which is introduced in this paper. 
The first variation of the internal energy density is

$$
\begin{aligned}
\delta U= & \sigma_{i j} \delta \gamma_{i j}+E_{k} \delta D_{k}-\sum_{I=1}^{N} \delta \rho_{I}\left(\sigma_{i j} \Delta \gamma_{i j}^{s(I)}\right. \\
& \left.+E_{k} \Delta D_{k}^{s(I)}+\Delta d_{k i j}^{(I)} \sigma_{i j} E_{k}\right) .
\end{aligned}
$$

The first variation of the total energy of the system is

$$
\begin{aligned}
\delta \Phi= & \int_{V} \delta U \mathrm{~d} V-\int_{S_{\sigma}} \bar{t}_{i} \delta u_{i} \mathrm{~d} S+\int_{S_{\phi}} \bar{\phi} \delta D_{i} n_{i} \mathrm{~d} S \\
& +\int_{V}\left(\sum_{I=1}^{N} \delta \rho_{I} W_{\mathrm{cr}}^{(I)}\right) \mathrm{d} V .
\end{aligned}
$$

Substitute Eq. (14) into Eq. (15), we get

$$
\begin{aligned}
\delta \Phi= & \int_{V}\left[\sigma_{i j} \delta \gamma_{i j}+E_{k} \delta D_{k}-\sum_{I=1}^{N} \delta \rho_{I}\left(\sigma_{i j} \Delta \gamma_{i j}^{s(I)}\right.\right. \\
& \left.\left.+E_{k} \Delta D_{k}^{s(I)}+\Delta d_{k i j}^{(I)} \sigma_{i j} E_{k}-W_{\mathrm{cr}}^{(I)}\right)\right] \mathrm{d} V \\
& -\int_{S_{\sigma}} \bar{t}_{i} \delta u_{i} \mathrm{~d} S+\int_{S_{\phi}} \bar{\phi} \delta D_{i} n_{i} \mathrm{~d} S .
\end{aligned}
$$

The displacement $u_{i}$ which satisfies the boundary condition (9) is called the kinematical admissible displacement and the electric displacement $D_{i}$ which satisfies the field Eq. (7) and the boundary condition (10) is called the electric admissible electric displacement. The volume fraction $\rho_{I}$ of the domain switching that satisfies the conditions $\rho_{I} \geq 0$ and $\sum_{I=1}^{N} \rho_{I}=1$ is called the physically admissible volume fraction.

We have the following principle of stationary total energy.

Among all kinematical admissible displacements $u_{i}$, electric admissible electric displacements $D_{i}$ and physically admissible volume fraction $\rho_{I}$, the actual displacement $u_{i}^{*}$, actual electric displacement $D_{i}^{*}$ and actual volume fraction $\rho_{I}^{*}$ make the total energy $\Phi$ stationary. The proof of the principle of stationary total energy is given in the Appendix A.

According to this principle of stationary total energy, one can obtain the stress field Eq. (6) and boundary conditions (8) and (11). In addition, one can also obtain the following switching criterion

$$
\begin{array}{r}
\sigma_{i j} \Delta \gamma_{i j}^{s(I)}+E_{k} \Delta D_{k}^{s(I)}+\Delta d_{k i j}^{(I)} \sigma_{i j} E_{k}=W_{\mathrm{cr}}^{(I)} \\
(I \in 1,2,3, \ldots, N) .
\end{array}
$$

It should be emphasized that the Eq. (17) is only available for the variant $I$ in which the domain switching is active (It means that the volume fraction $\rho_{I}$ satisfied the active condition $\rho_{I}>0$ ). Equation (17) is the modification and further development of the energy criterion proposed by Hwang et al. [1]. It accords with the work by Hannes and Herbert
[28] in the spirit. Substitute Eq. (5) into Eq. (17), a set of linear algebraic equations for $\rho_{I}$ are obtained

$\sum_{J=1}^{N} \rho_{J} A_{I J}=\hat{W}_{\mathrm{cr}}^{(I)} \quad(I \in 1,2,3, \ldots, N)$,

where

$$
\begin{aligned}
& A_{I J}=C_{i j k l} \Delta \hat{\gamma}_{i j}^{(J)} \Delta \hat{\gamma}_{k l}^{(I)}, \\
& \Delta \hat{\gamma}_{i j}^{(I)}=\Delta \gamma_{i j}^{s(I)}+\Delta d_{k i j}^{(I)} E_{k}, \quad(I \in 1,2,3, \ldots, N), \\
& \hat{W}_{\mathrm{cr}}^{(I)}=\hat{\sigma}_{i j} \Delta \hat{\gamma}_{i j}^{(I)}+E_{k} \Delta D_{k}^{s(I)}-W_{\mathrm{cr}}^{(I)}, \\
& (I \in 1,2,3, \ldots, N) \text {, } \\
& \hat{\sigma}_{m n}=C_{m n i j}\left(\gamma_{i j}-\gamma_{i j}^{s(0)}-d_{k i j}^{(0)} E_{k}\right) .
\end{aligned}
$$

It must be emphasized that the unknown quantities in the coefficients $A_{I J}$ are the strain and the electric field. Once the strain and electric field are given, the volume fraction $\rho_{I}$ of the domain switching can be determined by Eq. (18). Especially for plane problem, $\rho_{1}$ is the volume fraction of $180^{\circ}$ switching, $\rho_{2}$ is the volume fraction of clockwise $90^{\circ}$ switching, $\rho_{3}$ is the volume fraction of counter clockwise $90^{\circ}$ switching.

When the domain only switches by $180^{\circ}$, we have

$\rho_{1}=\frac{\hat{W}_{\mathrm{cr}}^{(1)}}{A_{11}}$

When the domain only switches by clockwise $90^{\circ}$, we have

$\rho_{2}=\frac{\hat{W}_{\mathrm{cr}}^{(2)}}{A_{22}}$.

When the domain only switches by counter clockwise $90^{\circ}$, we have

$\rho_{3}=\frac{\hat{W}_{\mathrm{cr}}^{(3)}}{A_{33}}$.

When the domain switches by both the $180^{\circ}$ and clockwise $90^{\circ}$, we have

$$
\begin{aligned}
& \rho_{1}=\frac{\hat{W}_{\mathrm{cr}}^{(1)} A_{22}-\hat{W}_{\mathrm{cr}}^{(2)} A_{12}}{A_{11} A_{22}-A_{12} A_{21}}, \\
& \rho_{2}=\frac{\hat{W}_{\mathrm{cr}}^{(2)} A_{11}-\hat{W}_{\mathrm{cr}}^{(1)} A_{21}}{A_{11} A_{22}-A_{12} A_{21}} .
\end{aligned}
$$

When the domain switches by both the $180^{\circ}$ and counter clockwise $90^{\circ}$, we have

$$
\begin{aligned}
& \rho_{1}=\frac{\hat{W}_{\mathrm{cr}}^{(1)} A_{33}-\hat{W}_{\mathrm{cr}}^{(3)} A_{13}}{A_{11} A_{33}-A_{13} A_{31}}, \\
& \rho_{3}=\frac{\hat{W}_{\mathrm{cr}}^{(3)} A_{11}-\hat{W}_{\mathrm{cr}}^{(1)} A_{31}}{A_{11} A_{33}-A_{13} A_{31}} .
\end{aligned}
$$


Fig. 1 Schematics of single domain mechanical model

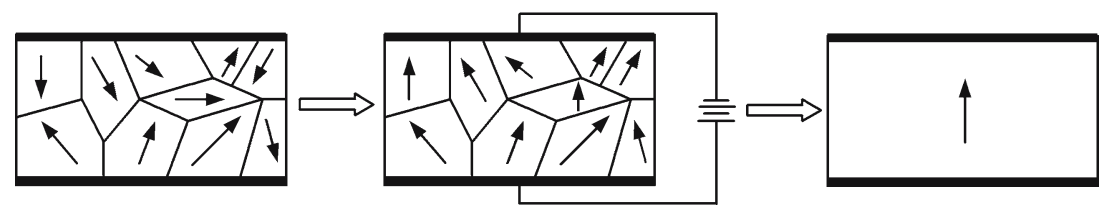

If the whole domain has completely switched, a partial domain switches by $90^{\circ}$ and the others switch by $180^{\circ}$. It means that

$\rho_{1}+\rho_{2}=1$.

It must be pointed out that the Eq. (30) is obtained when the mixture domain switching occurs by both $180^{\circ}$ and clockwise $90^{\circ}$. For this saturated situation of the mixture domain switching, the variation of the total energy is no longer the Eq. (16), and becomes

$$
\begin{gathered}
\sigma_{i j}\left(\Delta \gamma_{i j}^{s(2)}-\Delta \gamma_{i j}^{s(1)}\right)+E_{k}\left(\Delta D_{k}^{s(2)}-\Delta D_{k}^{s(1)}\right) \\
+\left(\Delta d_{k i j}^{(2)}-\Delta d_{k i j}^{(1)}\right) \sigma_{i j} E_{k}=\left(W_{\mathrm{cr}}^{(2)}-W_{\mathrm{cr}}^{(1)}\right) .
\end{gathered}
$$

From the first one of the Eq. (4), we can get

$$
\begin{aligned}
\sigma_{i j}=C_{i j m n}[ & \gamma_{m n}-\gamma_{m n}^{s(0)}-\left(d_{k m n}^{(0)}+\sum_{I=1}^{2} \rho_{I} \Delta d_{k m n}^{(I)}\right) E_{k} \\
& \left.-\sum_{I=1}^{2} \rho_{I} \Delta \gamma_{m n}^{s(I)}\right] .
\end{aligned}
$$

Substitute the Eq. (32) into the Eq.(31), we obtain

$$
\begin{gathered}
C_{i j m n}\left[\gamma_{m n}-\gamma_{m n}^{s(0)}-\left(d_{k m n}^{(0)}+\sum_{I=1}^{2} \rho_{I} \Delta d_{k m n}^{(I)}\right) E_{k}\right. \\
\left.-\sum_{I=1}^{2} \rho_{I} \Delta \gamma_{m n}^{s(I)}\right]\left(\Delta \gamma_{i j}^{s(2)}-\Delta \gamma_{i j}^{s(1)}\right) \\
+E_{k}\left(\Delta D_{k}^{s(2)}-\Delta D_{k}^{s(1)}\right)+C_{i j m n}\left(\Delta d_{k i j}^{(2)}-\Delta d_{k i j}^{(1)}\right) \\
\times E_{k}\left[\gamma_{m n}-\gamma_{m n}^{s(0)}-\left(d_{k m n}^{(0)}+\sum_{I=1}^{2} \rho_{I} \Delta d_{k m n}^{(I)}\right) E_{k}\right. \\
\left.-\sum_{I=1}^{2} \rho_{I} \Delta \gamma_{m n}^{s(I)}\right]=\left(W_{\mathrm{cr}}^{(2)}-W_{\mathrm{cr}}^{(1)}\right) .
\end{gathered}
$$

Then we have

$$
\rho_{2}=\frac{\hat{W}_{\mathrm{cr}}^{(2)}-\hat{W}_{\mathrm{cr}}^{(1)}-\left(A_{12}-A_{11}\right)}{\left(A_{11}+A_{22}\right)-A_{12}-A_{21}}, \quad \rho_{1}=1-\rho_{2} .
$$

\section{Mechanical model for ferroelectric specimen}

\subsection{Mechanical model}

In order to describe the electromechanical coupling phenomena of the ferroelectric specimen, the hardening relation between the driving force of domain switching and the volume fraction of domain switching need to be determined. A plane strain single mechanical model is proposed in this paper. As shown in Fig. 1, the poled ferroelectric specimen is considered as a transversely isotropic homogeneous material with single domain. The single domain is assumed to switch when the reduction of potential energy of the system due to that switch exceeds a critical value, which can be considered as the energy barrier that must be overcome to achieve the switch.

The switching criterion proposed by Hwang et al. [1] can be expressed as

$$
E_{i} \Delta D_{i}^{s(I)}+\sigma_{i j} \Delta \gamma_{i j}^{s(I)}=W_{\mathrm{cr}}^{(I)}
$$

The initial energy barrier against the $90^{\circ}$ switch is assumed to be $\frac{3}{2} \sigma_{c} \gamma^{s}$, the initial energy barrier against the $180^{\circ}$ switch is $2 E_{c} P_{s}, \sigma_{c}$ is the coercive stress, $\gamma^{s}$ is the spontaneous strain. The present model is similar to the mechanical model proposed by Li et al. [29]. But two different points between these two models need to be emphasized. Firstly, the energy barrier will increase when the volume fraction of the domain switching increases in the present model meanwhile the energy barrier will keep constant in their model. Secondly, the 180 domain switching can be occurred directly in the present model.

In Lynch's experimental work [30], Samples were cut to $10 \mathrm{~mm}$ cubes, polished, and electroded with sputtered $\mathrm{Au} / \mathrm{Pd}$. The sample is first subjected to electric field and then to uniaxial stress loading. The silicone oil bath prevents high voltage arcing. The sample is isolated from the test frame by two alumina blocks, one on top and one on bottom. A servohydraulic test frame applies the compressive stress and a high voltage amplifier applies the electric field.

One can see from the experiment results by Lynch [30] that the butterfly loop tails become narrow and short when the compressive stress increases. The narrow tails indicate that the coercive electric fields $E_{c}^{\sigma}$ reduce with the compressive stress increasing clearly. 
Suppose the volume fraction of $180^{\circ}$ switching is $\rho_{1}$. The volume fraction of clockwise $90^{\circ}$ switching can be expressed as $\rho_{2}$. The volume fraction of counter clockwise $90^{\circ}$ switching can be expressed as $\rho_{3}$. Due to the symmetry of the model, only clockwise $90^{\circ}$ switch is considered in this model. The whole process of domain switching contains four stages when the ferroelectric specimen is subjected to both the negative electric and compressive mechanical loading. First the volume fraction $\rho_{2}$ of $90^{\circ}$ switching increases when $90^{\circ}$ domain switching takes place. Then the $180^{\circ}$ switching occurs when the applied electric field reaches the coercive electric field $E_{c}^{\sigma}$, meanwhile the $\rho_{2}$ keeps constant since the driving force of domain switch for the second $90^{\circ}$ switching is less than the energy barrier. Third when $\rho_{1}+\rho_{2}$ reaches $1, \rho_{1}$ increases continually and $\rho_{2}$ decreases to keep $\rho_{1}+\rho_{2}=1$. Last the electric field is reduced to zero while the mechanical load keeps constant. According to this process of domain switching, the hardening relation between the driving force of domain switching and the volume fraction of domain switching can be calibrated by using the partial experimental data by Lynch [30].

(1) Purely mechanical loadings

According to the domain switching criterion, only $90^{\circ}$ domain switching occurs. Using the constitutive Eq. (4) one can be obtained

$\rho_{2}=\frac{D_{3}-D_{3}^{s(0)}-d_{333}^{(0)} \sigma_{33}}{\Delta D_{3}^{s(2)}+\Delta d_{333}^{(2)} \sigma_{33}}$,

where $\gamma_{33}$ is the experimental result for the ferroelectric specimen under purely mechanical loading.

(2) Purely electric loadings

The domain can switch by $90^{\circ}$ or $180^{\circ}$. Only the $90^{\circ}$ domain switch occurs before the applied electric field reaches the coercive electric field.

$\rho_{2}=\frac{\gamma_{33}-\gamma_{33}^{s(0)}-d_{333}^{(0)} E_{3}}{\Delta \gamma_{33}^{s(2)}+\Delta d_{333}^{(2)} E_{3}}$.

After the applied electric field is greater than the coercive electric field, the $180^{\circ}$ domain switching occurs. If $\rho_{1}+\rho_{2}$ is less than $1, \rho_{1}$ increases continually and $\rho_{2}$ keeps constant.

$\rho_{1}=\frac{\gamma_{33}-\gamma_{33}^{s(0)}-\rho_{2} \Delta \gamma_{33}^{s(2)}-\left(d_{333}^{(0)}+\rho_{2} \Delta d_{333}^{(2)}\right) E_{3}}{\Delta d_{333}^{(1)} E_{3}}$.

When $\rho_{1}+\rho_{2}$ reach $1, \rho_{1}$ increases continually and $\rho_{2}$ decreases according to following formula

$\rho_{1}=\frac{\gamma_{33}-\gamma_{33}^{s(0)}-\Delta \gamma_{33}^{s(2)}-\left(d_{333}^{(0)}+\Delta d_{333}^{(2)}\right) E_{3}}{\left(\Delta d_{333}^{(1)}-\Delta d_{333}^{(2)}\right) E_{3}-\Delta \gamma_{33}^{s(2)}}$,

$\rho_{2}=1-\rho_{1}$.
(3) Electromechanical loadings

After a prescribed compressive stress is applied to the specimen and held fixed, the strain of the specimen can be expressed as $\gamma_{33}^{(0)}$ and the volume fraction of $90^{\circ}$ domain switching is $\rho_{2}^{(0)}$. Then a circle of electric field is introduced. The strain increment is

$$
\begin{aligned}
\Delta \gamma_{33}= & \gamma_{33}-\gamma_{33}^{(0)}=\left(\rho_{2}-\rho_{2}^{(0)}\right) \Delta \gamma_{33}^{s(2)} \\
& +\left(d_{333}^{(0)}+\rho_{1} \Delta d_{333}^{(1)}+\rho_{2} \Delta d_{333}^{(2)}\right) E_{3} .
\end{aligned}
$$

Only the $90^{\circ}$ domain switch occurs when the applied electric field is less than the coercive electric field $E_{c}^{\sigma}$.

$\rho_{2}=\frac{\Delta \gamma_{33}+\rho_{2}^{(0)} \Delta \gamma_{33}^{s(2)}-d_{333}^{(0)} E_{3}}{\Delta \gamma_{33}^{s(2)}+\Delta d_{333}^{(2)} E_{3}}$.

After the applied electric field is greater than the coercive electric Field $E_{c}^{\sigma}$, the $180^{\circ}$ domain switch occurs. If $\rho_{1}+\rho_{2}$ is less than $1, \rho_{1}$ increases continually and $\rho_{2}$ keeps constant.

$\rho_{1}=\frac{\Delta \gamma_{33}-\left(\rho_{2}-\rho_{2}^{(0)}\right) \Delta \gamma_{33}^{s(2)}-\left(d_{333}^{(0)}+\rho_{2} \Delta d_{333}^{(2)}\right) E_{3}}{\Delta d_{333}^{(1)} E_{3}}$.

When $\rho_{1}+\rho_{2}$ reaches $1, \rho_{1}$ increases continually and $\rho_{2}$ decreases.

$\rho_{1}=\frac{\Delta \gamma_{33}-\left(1-\rho_{2}^{(0)}\right) \Delta \gamma_{33}^{s(2)}-\left(d_{333}^{(0)}+\Delta d_{333}^{(2)}\right) E_{3}}{\left(\Delta d_{33}^{(1)}-\Delta d_{333}^{(2)}\right) E_{3}-\Delta \gamma_{33}^{s(2)}}$,

$\rho_{2}=1-\rho_{1}$.

\subsection{The hardening relation}

According to the partial experimental results, one can easily obtain the measurement value $\gamma_{33}$ or $D_{3}$ when the ferroelectric specimen is subjected to different stress and electric fields. Substituting the stress and electric field and the corresponding $\gamma_{33}$ or $D_{3}$ into Eqs. (36)-(43), the hardening relation between the driving force of the domain switching and the volume fraction of the domain switching can be calibrated. Then the relation can be dimensionless by the initial energy barrier,

$f_{1}\left(\rho_{1}\right)=\frac{W_{\mathrm{cr}}^{(1)}}{2 E_{c} P_{s}}$,
$f_{2}\left(\rho_{2}\right)=\frac{W_{\mathrm{cr}}^{(2)}}{1.5 \sigma_{c} \gamma_{s}^{s}}$.

In this paper for the ferroelectric single domain (PLZT 8/65/35) the material parameters are chosen to be $Y=62 \times 10^{9} \mathrm{~Pa}, v=0.3, \varepsilon_{33}=62 \times 10^{-9} \mathrm{C} /(\mathrm{V} \mathrm{m})$, $\gamma_{s}=0.143 \times 10^{-2}, P_{s}=0.25 \mathrm{C} / \mathrm{m}^{2}, E_{c}=0.36 \mathrm{MV} / \mathrm{m}$, $d_{333}=0.9 \times 10^{-9} \mathrm{~m} / \mathrm{V}, d_{31}=-4.5 \times 10^{-10} \mathrm{~m} / \mathrm{V}, d_{15}=$ $1.35 \times 10^{-9} \mathrm{~m} / \mathrm{V}$. According to the experimental data measured by Lynch, the corresponding "coercive electric fields" 


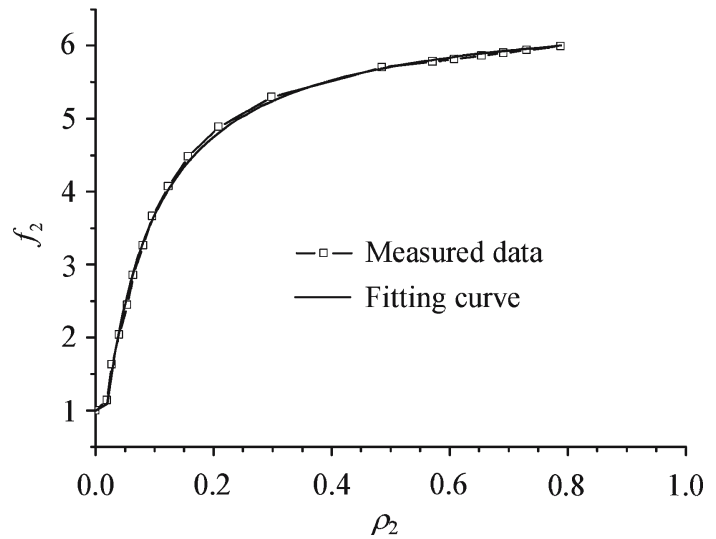

Fig. 2 The hardening relation and fitted hardening function between $f_{2}$ and $\rho_{2}$ under purely electric loading

$E_{c}^{\sigma}$ of ferroelectric ceramics are $0.36,0.2,0.14,0.075 \mathrm{MV} / \mathrm{m}$ under a uniaxial compressive stress $0,-15,-30,-60 \mathrm{MPa}$.

The experimental data taken in the following analyses are on the basis of the Lynch's work [30] for ferroelectric ceramics PLZT 8/65/35.

First we discuss $90^{\circ}$ domain switching:

(1) Purely electric loadings

The hardening relation curve of $90^{\circ}$ domain switching between the driving force of domain switching and the volume fraction of domain switching is shown in Fig. 2.

The fitting hardening function of $90^{\circ}$ domain switch is given by

$f_{2}\left(\rho_{2}\right)=1+\frac{1.289\left(1-\mathrm{e}^{-2.588 \rho_{2}}\right)}{\left(1-\mathrm{e}^{-2.588}\right)}+\frac{3.711\left(1-\mathrm{e}^{-15.47 \rho_{2}}\right)}{\left(1-\mathrm{e}^{-15.47}\right)}$.

(2) Purely mechanical loadings

The hardening relation of $90^{\circ}$ domain switch between the driving force of domain switching and the volume fraction of domain switching is shown in Fig. 3.

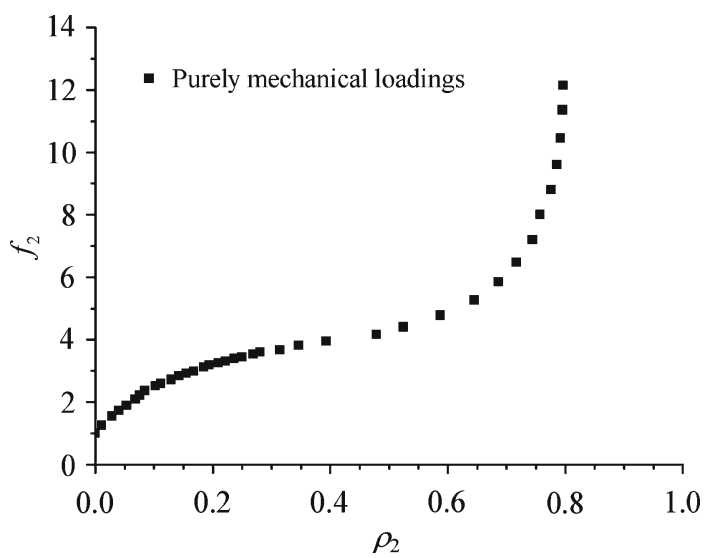

Fig. 3 The hardening relation between $f_{2}$ and $\rho_{2}$ under purely mechanical loadings

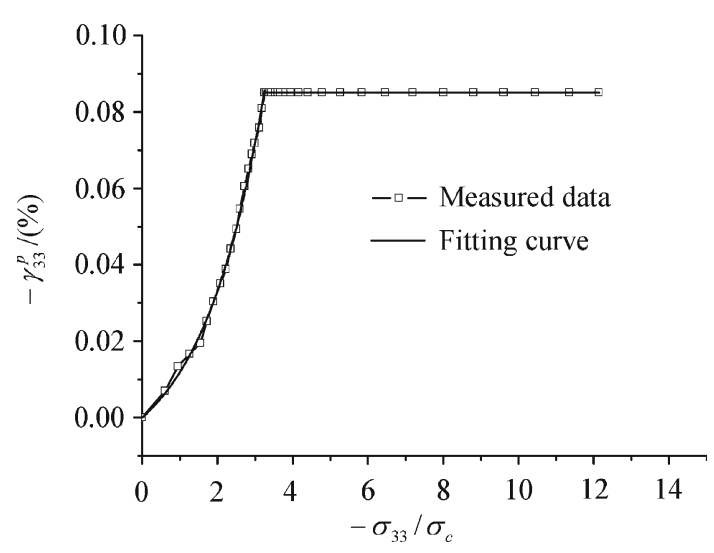

Fig. 4 The relationship and fitted curves between $\gamma_{33}^{p}$ and $-\sigma_{33} / \sigma_{c}$

The ferroelectric ceramics usually contains many micro pores. The irreversible strain, which is called "plastic" strain, denoted as $\gamma_{33}^{p}$, will be generated under compressive loading due to the pores. According to the experimental results measured by Lynch [30], one can obtain the following fitting formula

$$
\begin{aligned}
\gamma_{33}^{p}= & 0.01477\left(\mathrm{e}^{-0.5856 \sigma_{33} / \sigma_{c}}-1\right), \\
& -\sigma_{33} / \sigma_{c}<3.26 .
\end{aligned}
$$

It must be pointed out that the formula (47) is only suitable for $-\sigma_{33} / \sigma_{c}<3.26$. When $-\sigma_{33} / \sigma_{c} \geq 3.26$, these pores will collapse so that the plastic strain keeps constant. Hence the plastic strain $\gamma_{33}^{p}$ is equal to $-0.085 \%$ for $-\sigma_{33} / \sigma_{c} \geq 3.26$ (Fig. 4).

(3) Electromechanical coupling loadings

The hardening relation curves of $90^{\circ}$ domain switch under electromechanical coupling loadings are shown in Fig. 5. The hardening curves of $90^{\circ}$ domain switch under electromechanical coupling loadings are quite similar to that of purely mechanical loading. The comparison between Figs. 3 and 5 is shown in Fig. 6. A single function is used to fit the similar hardening relation in two loading conditions. The function can be expressed as

$$
f_{2}\left(\rho_{2}\right)=\frac{7.340 \rho_{2}}{0.2790+\rho_{2}}+\frac{1.835 \rho_{2}}{0.9799-\rho_{2}}-6.256 \rho_{2}+1 .
$$

The comparison between the fitting function (48) and the experimental data for the purely mechanical loading is given in Fig. 7.

Now we discuss $180^{\circ}$ domain switch.

(4) Purely electric loadings

The hardening relation of 180 domain switch is shown in Fig. 8.

The corresponding fitting function is

$$
f_{1}\left(\rho_{1}\right)=1+0.0242 \rho_{1} /\left(1.043-1.024 \rho_{1}\right) .
$$




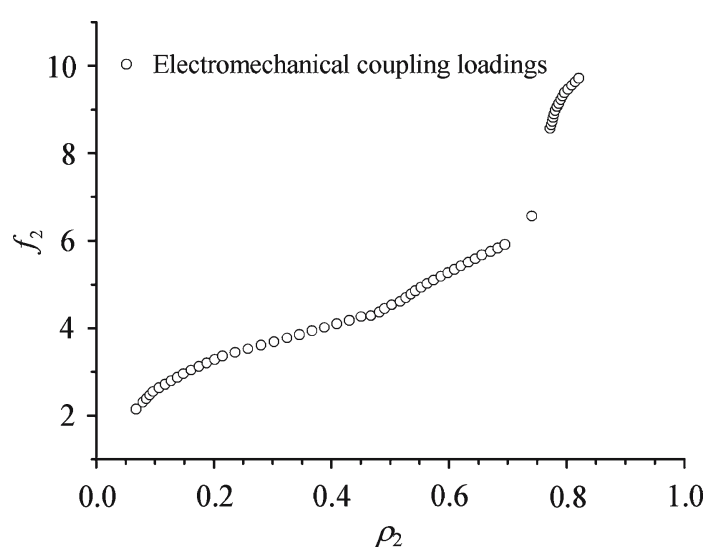

Fig. 5 The hardening relation between $f_{2}$ and $\rho_{2}$ under combined electromechanical loadings

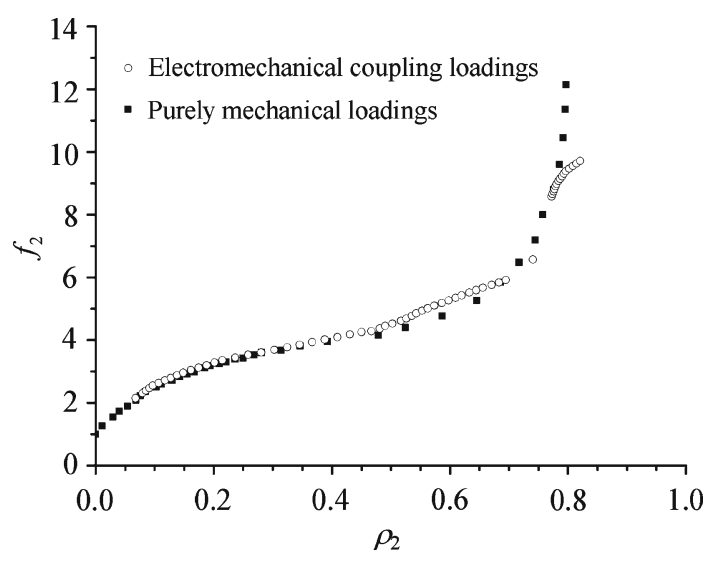

Fig. 6 Comparison of the hardening relation between combined electromechanical loadings and mechanical loadings

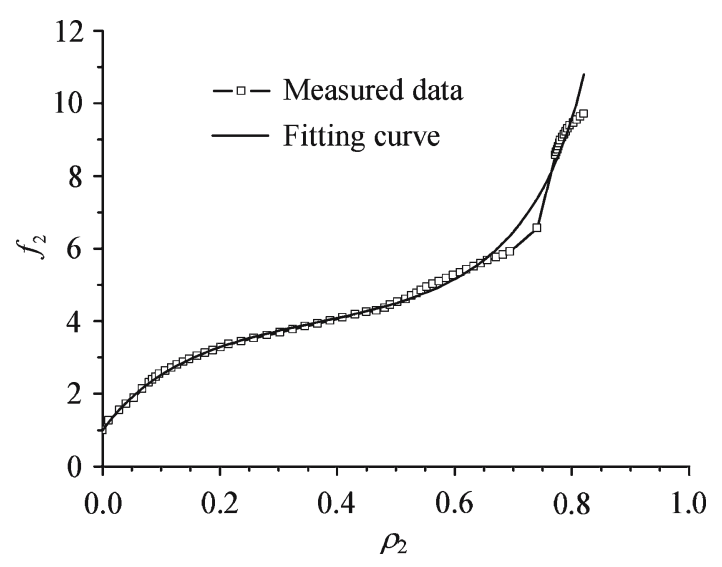

Fig. 7 The hardening relation between $f_{2}$ and $\rho_{2}$ under electromechanical coupling loadings and purely mechanical loadings

(5) Combined electromechanical loadings

The hardening relation curves of 180 domain switching between the driving force and the volume fraction of domain switching are shown in Fig. 9. The experimental data is complex. A linear function is used to fit the experimental data, as shown in Fig. 10.

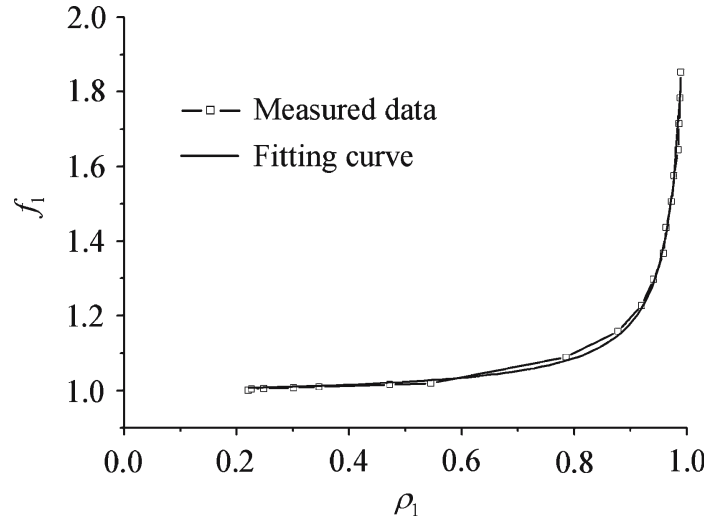

Fig. 8 The hardening relation between $f_{2}$ and $\rho_{2}$ under purely electric loadings

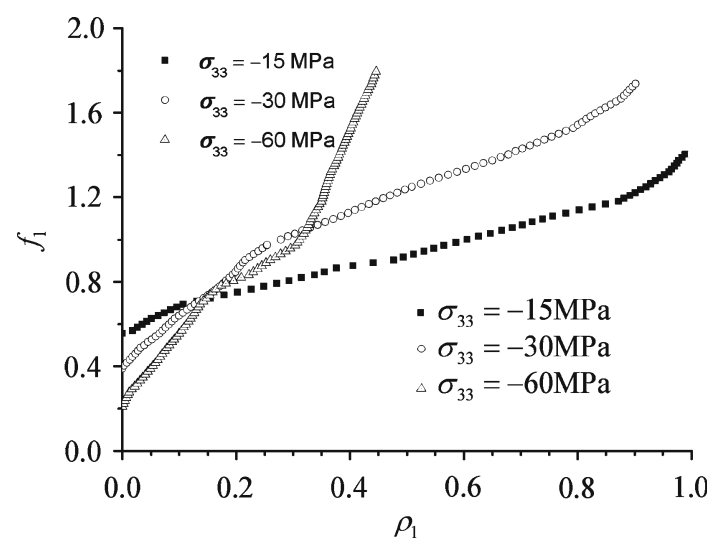

Fig. 9 The hardening relation between $f_{1}$ and $\rho_{1}$

The fitted function is

$f_{1}\left(\rho_{1}\right)=E_{c}^{\sigma} / E_{c}+B \cdot \rho_{1}$.

where $E_{c}^{\sigma}$ is the corresponding coercive electric field of ferroelectric specimen under a suppressive stress $\sigma$. Figure 11 shows that the slope $B$ versus corresponding stress $\sigma$ displays linear relation. The fitting function is

$B=0.0242-0.3607 \sigma_{33} / \sigma_{c}$.

The formula (49) for the purely electric loading and the formula (50) for the electromechanical loadings can be combined as a single formula as following

$f_{1}\left(\rho_{1}\right)=E_{c}^{\sigma} / E_{c}+B \cdot \rho_{1} \cdot H\left(-\sigma_{33} / \sigma_{c}\right)$,

where

$H\left(-\sigma_{33} / \sigma_{c}\right)=\left\{\begin{array}{cc}\frac{1}{1.043-1.024 \rho_{1}} & \sigma_{33}=0, \\ 1 & \sigma_{33} \neq 0 .\end{array}\right.$

When the electric unloading occurs after the negative electric load arrives the maximum value, the $90^{\circ}$ and $180^{\circ}$ domain switching cannot occur under purely mechanical loadings 


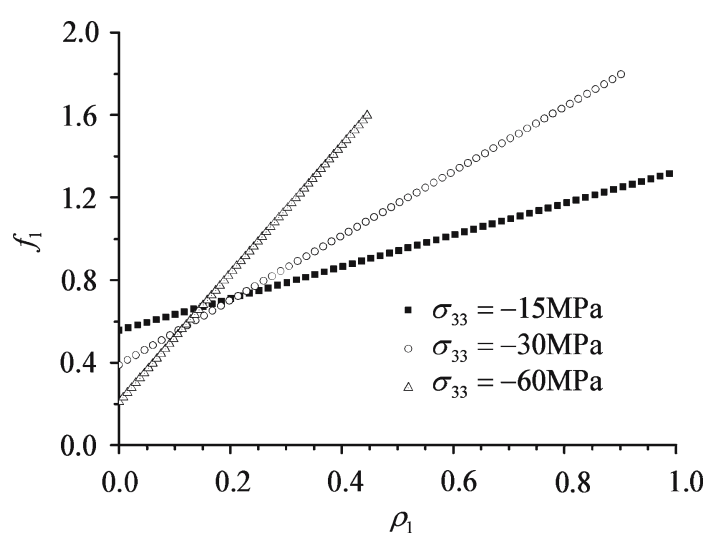

Fig. 10 The fitted hardening function between $f_{1}$ and $\rho_{1}$

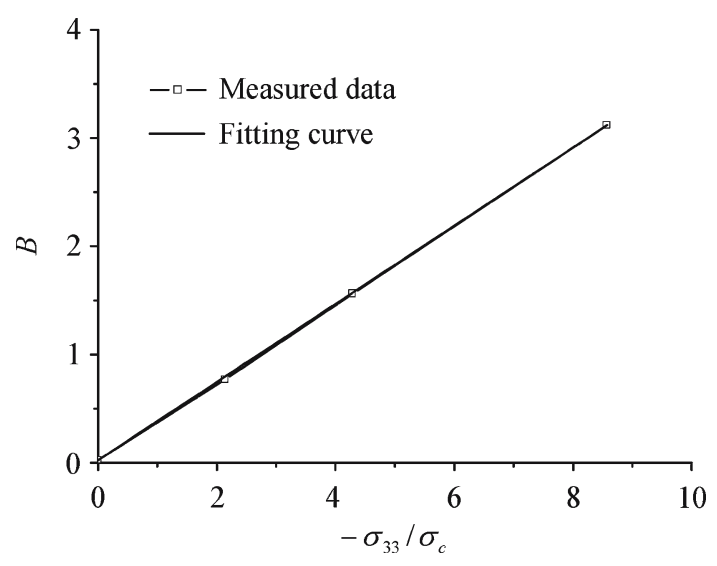

Fig. 11 The relation between $B$ and $-\sigma_{33} / \sigma_{c}$

and purely electric loadings since the driving force decreases. So the constitutive behavior is linear. As for combined electromechanical loadings, the $180^{\circ}$ domain switch cannot occur. But the $90^{\circ}$ domain switch will take place when the driving force of domain switch is greater than the value of hardening function. Then the volume fraction of $90^{\circ}$ switching is calculated according to Eq. (48).

\section{The calculation results}

According to the hardening relation between the driving force and the volume fraction of domain switching presented in the previous section, the nonlinear constitutive behavior of ferroelectric ceramics is calculated under three loading conditions.

\subsection{Uniaxial mechanical loading}

The nonlinear constitutive behavior of the ferroelectric body subjected to a purely uniaxial compressive stress is studied in this section. The comparison of simulated and measured results is shown in Figs. 12 and 13. The solid lines in Fig. 12

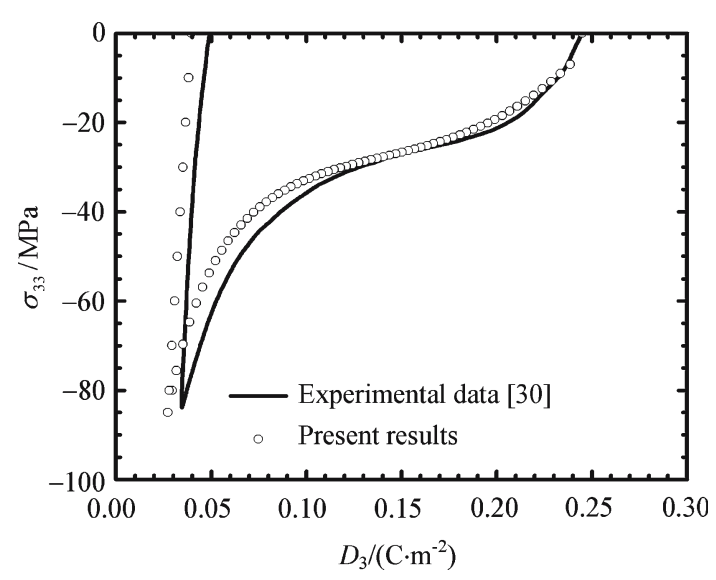

Fig. 12 Simulated and measured stress versus electric displacement at zero applied electric field

and Fig. 13 correspond to the experimental results by Lynch [30] and the present calculation results are plotted as the open circles.

Divide the load $-85 \mathrm{MPa}$ into 100 steps. The external mechanical loading is increased step by step. The behaviors of the stress versus electric displacement and stress versus strain are linear when the applied compressive stress is less than the critical value $\sigma_{c}$. In our simulation there is no domain switching before the applied compressive stress less than the critical value $\sigma_{c}$. The initial slope of the stress-electric displacement curve is the piezoelectric coefficient $d_{33}$. The initial slope of the stress-strain curve is the Young's module. The $90^{\circ}$ switching takes place when the uniaxial compressive stress exceeds the critical value $\sigma_{c}$. Now we discuss the unloading process from $\sigma_{33}=-85 \mathrm{MPa}$. In our simulation no domain switching occurs during unloading. Hence the behavior of the stress-electric displacement and stress-strain becomes linear again. The slope of the stress-electric displacement curve is close to infinity ( $d_{33}$ is nearly zero.) and the slope of the stress-strain curve is Young's modules again during unloading. Figure 12 shows that the calculated electric displacement versus applied compressive stress curve for the specimen is consistent with the experimental results for the case $\left|\sigma_{33}\right|<30 \mathrm{MPa}$. When $\sigma_{33}$ is less than $-30 \mathrm{MPa}$ the calculated results begin to deviate the experimental curve. At $-85 \mathrm{MPa}$ the calculated electric displacement $D_{3}$ is great than the experimental result. Figure 13 shows the axial stressstrain curve. When $\left|\sigma_{33}\right|$ is less than $30 \mathrm{MPa}$, the calculated results are in good agreement with the experimental results. With the increasing of the compressive stress the calculated axial strain $\gamma_{33}$ deviate the experimental curve gradually.

\subsection{Uniaxial electric field loading}

Figures 14, and 15 show the ferroelectric hysteresis and butterfly shaped curve under the uniaxial electric loading, 


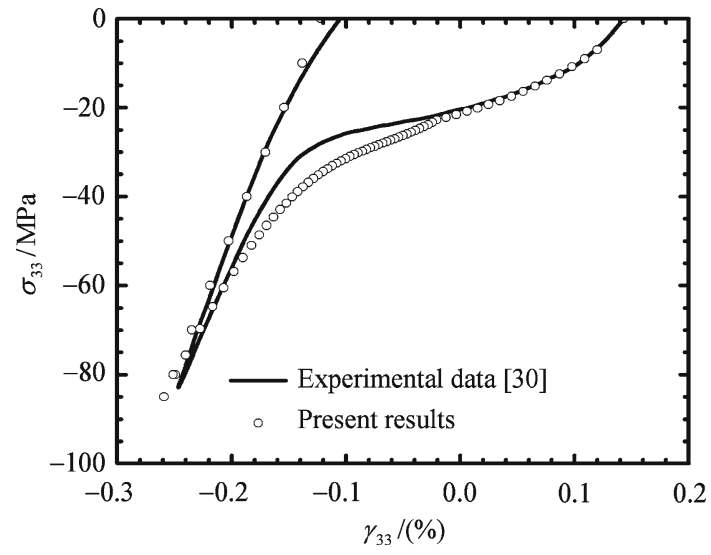

Fig. 13 Simulated and measured stress versus strain at zero applied electric field

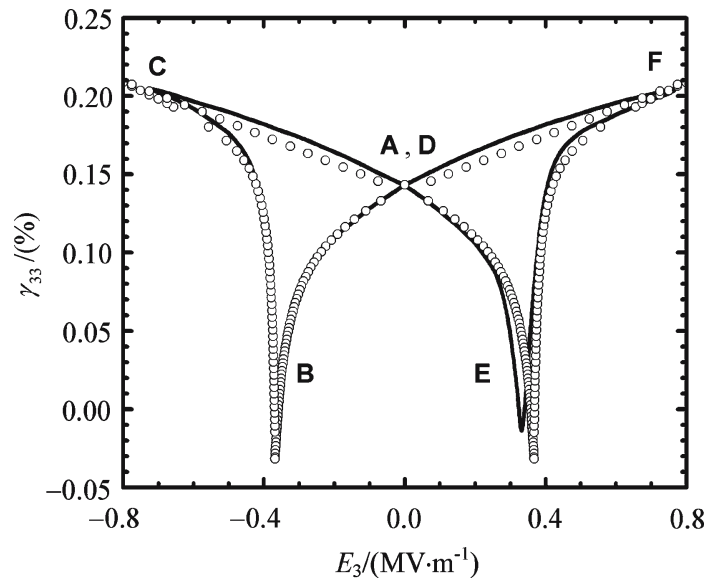

Fig. 14 Comparison of simulated and measured strain versus electric field curves at $\sigma_{33}=0$

respectively. First we discuss the negative electric field loading ( $E_{3}$ opposite to the direction of the remanent polarization of the specimen).

We can see from Fig. 14 that the calculated axial strain $\gamma_{33}$ agrees well with the experimental results from point $\mathrm{A}$ to points $\mathrm{B}$ and $\mathrm{C}$.

As shown in Fig. 15 the calculated electric displacement $D_{3}$ is in good agreement with the experimental results before $E_{c}$. Now a positive electric field is applied to the specimen from point $\mathrm{D}$.

When the electric field $E_{3}$ increases, the domain switching occurs in some elements. The situation of the domain switching is similar to that of the negative electric loading from point A to points B and C. The computed strain $\gamma_{33}$ and electric displacement $D_{3}$ for the specimen agree well with the experimental data from point $\mathrm{E}$ to point $\mathrm{F}$. The computed strain $\gamma_{33}$ and electric displacement $D_{3}$ for the specimen match well with the experimental data from point $\mathrm{F}$ and point A.

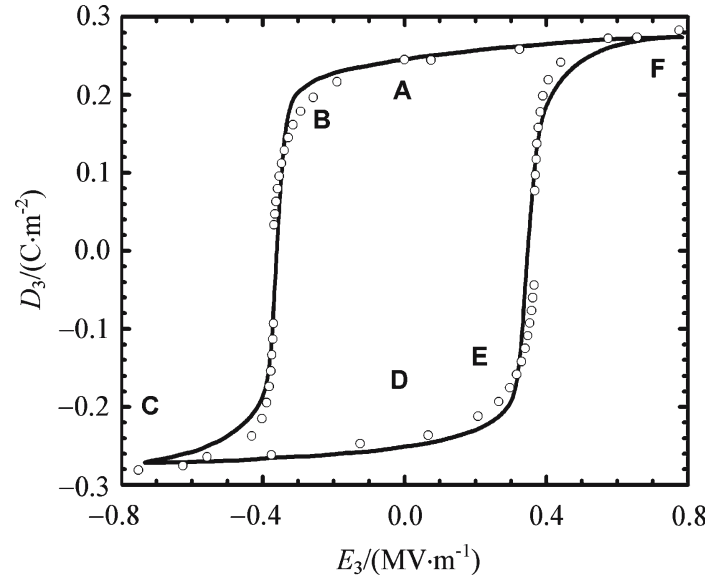

Fig. 15 Comparison of simulated and measured electric displacement versus electric field curves at $\sigma_{33}=0$

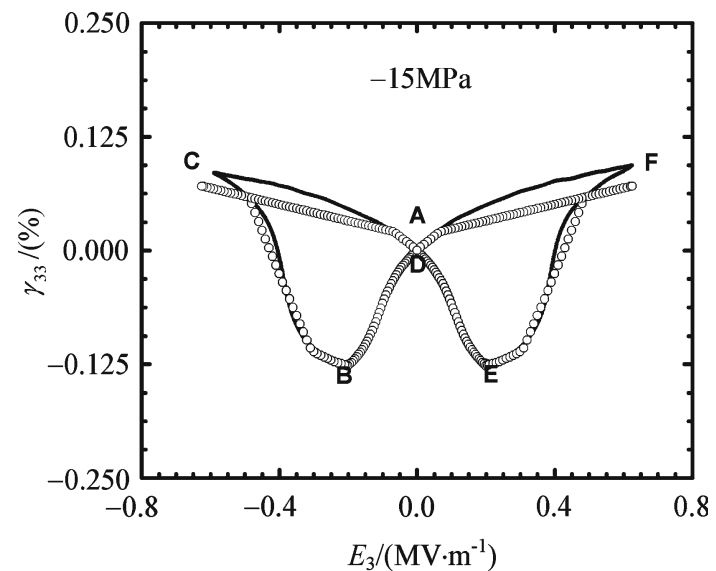

Fig. 16 Comparison of simulated and measured strain versus electric field curves at $\sigma_{33}=-15 \mathrm{MPa}$

\subsection{Combined electromechanical loading}

As for electromechanical coupling loading, first a prescribed compressive stress is applied to the specimen. Then a circle of electric field is applied to the specimen while the external compressive stress is held fixed. Figures 16, 17, 18, 19, 20, 21 show the results of experimental and calculated electric displacement and strain versus the applied electric field with different compressive stress loading. The solid lines in Figs. 16, 17, 18, 19, 20, 21 correspond to the experimental results obtained by Lynch [30] and the present calculation results are plotted as the open circles.

Now we discuss the case of $\sigma_{33}=-15 \mathrm{MPa}$. This prescribed compressive stress is applied to the specimen and held fixed. An electric parallel to the applied stress is then introduced and cycled between positive and negative limits. The general feature of the hysteresis loop and the butterfly loop for a constant stress of $-15 \mathrm{MPa}$ are captured quite well in the predicted curves. 


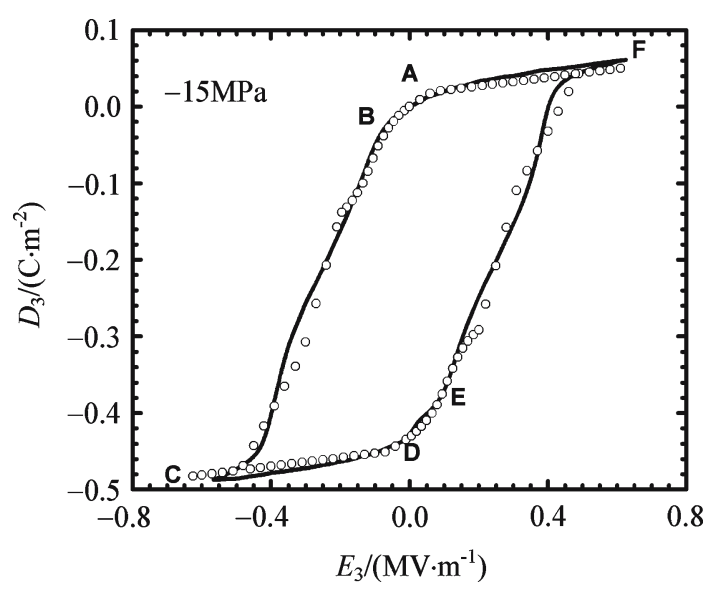

Fig. 17 Comparison of simulated and measured electric displacement versus electric field curves at $\sigma_{33}=-15 \mathrm{MPa}$

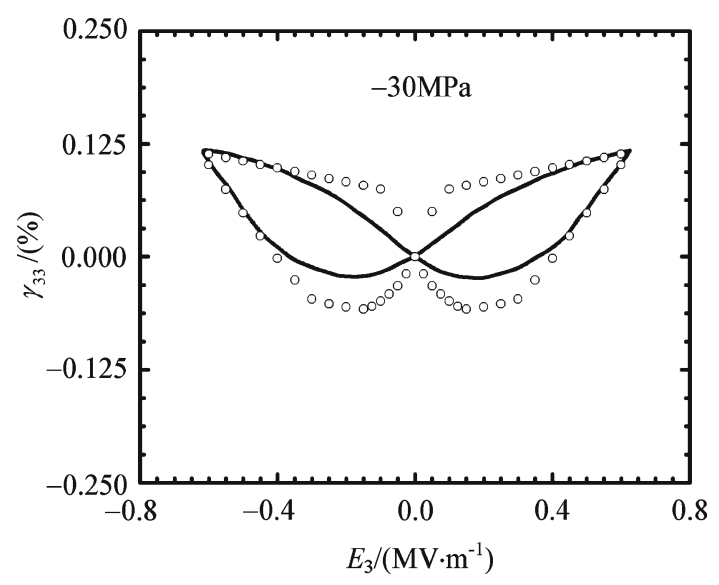

Fig. 18 Comparison of simulated and measured strain versus electric field curves at $\sigma_{33}=-30 \mathrm{MPa}$

For the cases of $\sigma_{33}=-30 \mathrm{MPa}$ and $-60 \mathrm{MPa}$, the comparison of simulated and measured longitudinal strain versus electric field is satisfying. But the remanent polarization is predicted relatively poorly in the calculated curves. This discrepancy is probably associated with deficiency of the model in handling $180^{\circ}$ switching. A simple linear fitting of hardening relation of $180^{\circ}$ switching cannot reflect the actual evolution of the domain switching.

\section{Conclusions}

According to the physical mechanism the volume fraction of domain switching is introduced in this paper. The minimum energy principle were put forward in which the basic unknown quantities are the displacement $u_{i}$, electric displacement $D_{i}$ and volume fraction $\rho_{I}$ of the domain switching for the variant $I$. Mechanical field equation and a domain switching criterion were deduced from the energy principle.

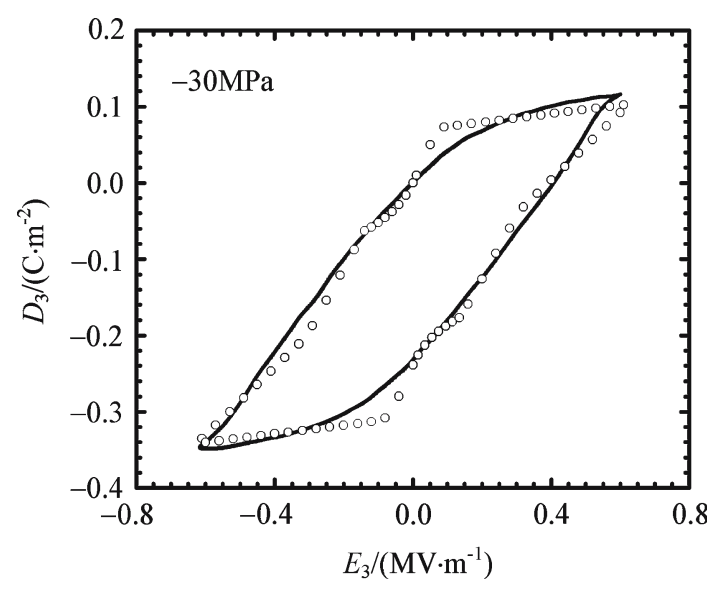

Fig. 19 Comparison of simulated and measured electric displacement versus electric field curves at $\sigma_{33}=-30 \mathrm{MPa}$

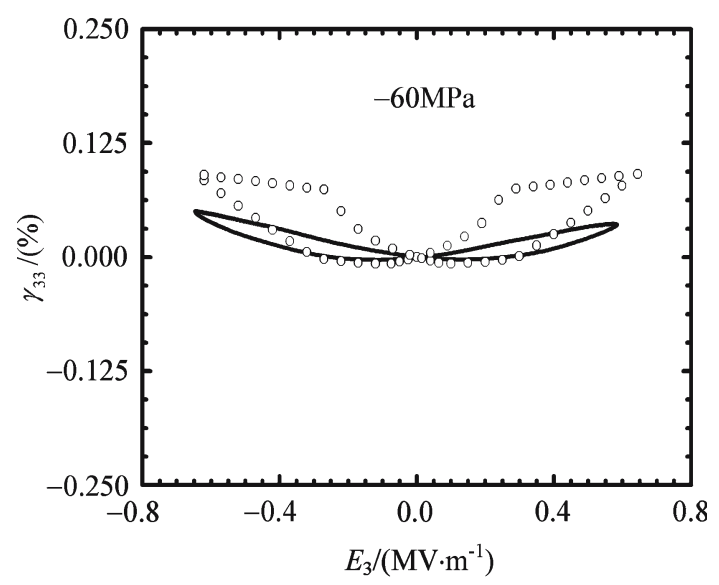

Fig. 20 Comparison of simulated and measured strain versus electric field curves at $\sigma_{33}=-60 \mathrm{MPa}$

The domain switching criterion established in this paper is the expansion and development of the energy criterion established by Hwang et al. [1]. According to the domain switching criterion, a set of linear algebraic equations for the volume fraction $\rho_{I}$ of the domain switching are obtained, which only contains the unknown strain and electric field. Furthermore, the single-domain mechanical model is developed in this paper. By using the partial experimental results, the hardening relation between the driving force of domain switching and the volume fraction of domain switching can be calibrated. The validity of the model is examined by means of the comparison of the simulations and experimental data. The calculation results are in good agreement with the experimental results. Recently researchers have more interest to characterise the multi-axial constitutive behaviors of ferroelectric ceramics. Huber and Fleck [6], Fang et al. [31] and Wang et al. [32] reported their experiments results of multi-axial behavior for ferroelectric materials. Experiments results by Wang et al. [32] show that the pre-stress when applied along the 


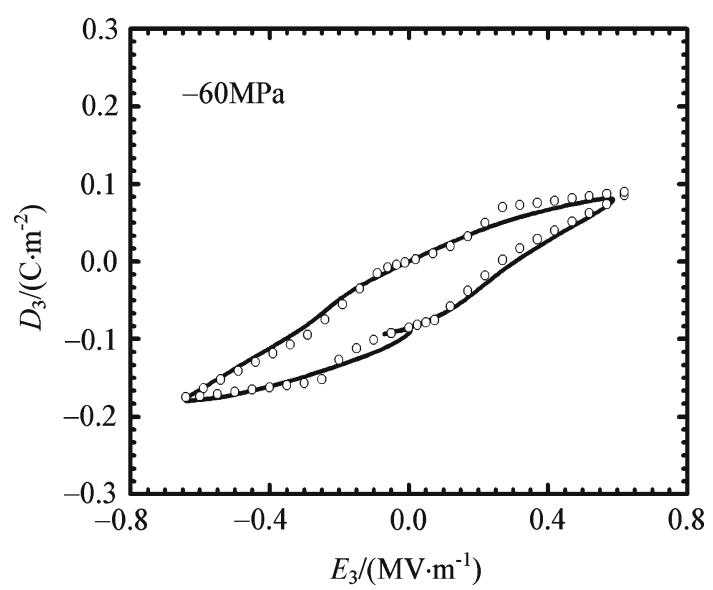

Fig. 21 Comparison of simulated and measured electric displacement versus electric field curves at $\sigma_{33}=-60 \mathrm{MPa}$

poling direction has a significant effect on electromechanical behavior of PZT53 while the pre-stress in a direction transverse to the poling direction has negligible effect on the electric hysteresis loops, dielectric coefficient and coercive electric field. So the next goal is the devolvement of present models capable of predicting multi-axial constitutive behaviors of ferroelectric ceramics.

\section{Appendix A}

The total energy $\Phi$ can be expressed as

$$
\begin{aligned}
\Phi= & \int_{V} U \mathrm{~d} V-\int_{S_{\sigma}} \bar{t}_{i} u_{i} \mathrm{~d} S+\int_{S_{\phi}} \bar{\phi} D_{i} n_{i} \mathrm{~d} S \\
& +\int_{V}\left(\sum_{I=1}^{N} \rho_{I} W_{\mathrm{cr}}^{(I)}\right) \mathrm{d} V .
\end{aligned}
$$

By using Lagrange multiplier $\lambda$, the principle of stationary total energy $\Phi$ is equivalent to the following principle of stationary functional $\tilde{\Phi}$ :

$$
\tilde{\Phi}=\Phi-\int_{V} \lambda D_{i, i} \mathrm{~d} V .
$$

The first variation of $\tilde{\Phi}$ has the form

$$
\begin{aligned}
\delta \tilde{\Phi}= & \int_{V}\left(\sigma_{i j} \delta \gamma_{i j}+E_{i} \delta D_{i}\right) \mathrm{d} V-\int_{S_{\sigma}} \bar{t}_{i} \delta u_{i} \mathrm{~d} S \\
& +\int_{S_{\phi}} \bar{\phi} n_{i} \delta D_{i} \mathrm{~d} S+\int_{V}\left(\sum_{I=1}^{N} \delta \rho_{I} W_{\mathrm{cr}}^{(I)}\right) \mathrm{d} V \\
& -\int_{V} D_{i, i} \delta \lambda \mathrm{d} V-\int_{V} \lambda \delta D_{i, i} \mathrm{~d} V .
\end{aligned}
$$

Above equation can be rewritten as

$$
\begin{aligned}
\delta \tilde{\Phi}= & \int_{V}\left[-\sigma_{i j, j} \delta u_{i}+\left(E_{i}+\lambda, i\right) \delta D_{i}\right. \\
& -\sum_{I=1}^{N} \delta \rho_{I}\left(\sigma_{i j} \Delta \gamma_{i j}^{s(I)}+E_{k} \Delta D_{k}^{s(I)}+\Delta d_{k i j}^{(I)} \sigma_{i j} E_{k}\right. \\
& \left.\left.-W_{\mathrm{cr}}^{(I)}\right)\right] \mathrm{d} V+\int_{S_{\sigma}}\left(\sigma_{i j} n_{j}-\bar{t}_{i}\right) \delta u_{i} \mathrm{~d} S \\
& -\int_{S_{\phi}}(\lambda-\bar{\phi}) n_{i} \delta D_{i} \mathrm{~d} S-\int_{V} D_{i, i} \delta \lambda \mathrm{d} V .
\end{aligned}
$$

The necessary conditions for $\tilde{\Phi}$ to be stationary is

$\delta \tilde{\Phi}=0$.

From Eq. (A.5), one can obtain

$\left\{\begin{array}{c}\sigma_{i j, j}=0, \\ E_{i}=-\lambda, i, \quad \text { in } V \\ D_{i, i}=0\end{array}\right.$

$\sigma_{i j} \Delta \gamma_{i j}^{s(I)}+E_{k} \Delta D_{k}^{s(I)}+\Delta d_{k i j}^{(I)} \sigma_{i j} E_{k}$
$=W_{\mathrm{cr}}^{(I)}, \quad$ in $V$,

$\sigma_{i j} n_{j}=\bar{t}_{i}, \quad$ on $S_{\sigma}$,

$\lambda=\bar{\phi}, \quad$ on $S_{\phi}$.

Clearly these are the basic equations and the boundary conditions for the ferroelectric body. From Eq. (A.6) and Eq. (A.9) one can see that the Lagrange multiply $\lambda$ is the electric potential $\phi$.

\section{References}

1. Hwang, S.C., Lynch, C.S., McMeeking, R.M.: Ferroelectric/ferroelastic interactions and a polarization switching model. Acta Metall. Mater. 43, 2073-2084 (1995)

2. Lu, W., Fang, D.N., Hwang, K.C.: Nonlinear electric-mechanical behavior and micormechanics modeling of ferroelectric domain evolution. Acta Mater. 47, 2913-2926 (1999)

3. Chen, X., Fang, D.N., Hwang, K.C.: Micromechanics simulation of ferroelectric polarization switching. Acta Mater. 45, 31813189 (1997)

4. Huo, Y., Jiang, Q.: Modeling of domain switching in ferroelektric ceramics: an example. Int. J. Solids Struct. 35, 1339-1353 (1998)

5. Huber, J.E., Fleck, N.A., Landis, C.M., McMeeking, R.M.: A constitutive model for ferroelectric polycrystals. J. Mech. Phys. Solids 47, 1663-1697 (1999)

6. Huber, J.E., Fleck, N.A.: Multi-axial electrical switching of a ferroelectric: theory versus experiment. J. Mech. Phys. Solids 49, 785-811 (2001) 
7. Huber, J.E., Fleck, N.A.: Ferroelectric switching: a micromechanics model versus measured behaviour. Eur. J. Mech. A/Solids 23, 203-217 (2004)

8. Hwang, S.C., Huber, J.E., McMeeking, R.M., Fleck, N.A.: The simulation of switching in polycrystalline ferroelectric ceramics. J. Appl. Phys. 84, 1530-1540 (1998)

9. Chen, W., Lynch, C.S.: A micro-electro-mechanical model for polarization switching of ferroelectric materials. Acta Mater. 46, 5303-5311 (1998)

10. Chen, W., Lynch, C.S.: Finite element analysis of cracks in ferroelectric ceramic materials. Eng. Fract. Mech. 64, 539-562 (1999)

11. Steinkopff, T.: Finite-element modeling of ferroelectric domain switching in piezoelectric ceramics. J. Eur. Ceramic Soc. 19, 1247-1249 (1999)

12. Chen, P.J., Peercy, P.S.: One dimensional dynamic electromechanical constitutive relations of ferroelectric materials. Acta Mech. 31, 231-241 (1979)

13. Chen, P.J.: Three dimensional dynamic electromechanical constitutive relations for ferroelectric materials. Int. J. Solids Struct. 16, 1059-1067 (1980)

14. Chen, P.J., Madsen, M.M.: One dimensional polar response of the electrooptic PLZT $7 / 65 / 35$ due to domain switching. Acta Mech. 41, 255-264 (1981)

15. Bassiouny, A.F., Ghaleb, G., Maugin, G.: Thermodynamical formulation for coupled electromechanical hysteresis effects-I basic equations. Int. J. Eng. Sci. 26, 1279-1295 (1988)

16. Bassiouny, A.F., Ghaleb, G., Maugin, G.: Thermodynamical formulation for coupled electromechanical hysteresis effects-II poling of ceramics. Int. J. Eng. Sci. 26, 1297-1306 (1988)

17. Kamlah, M., Tsakmakis, C.: Phenomenological modeling of the non-linear electromechanical coupling in ferroelectrics. Int. J. Solids Struct. 36, 669-695 (1999)

18. Kamlah, M., Böhle, U.: Finite element analysis of piezoceramics components taking into account ferroelectric hysteresis behavior. Int. J. Solids Struct. 38, 605-633 (2001)

19. Kamlah, M., Wang, Z.: A thermodynamically and microscopically motivated constitutive model for piezo-ceramics. Comput. Mater. Sci. 28, 409-418 (2003)
20. Cocks, A.C.F., McMeeking, R.M.: A phenomenological constitutive law for the behavior of ferroelectric ceramics. Ferroelectrics 228, 219-228 (1999)

21. Landis, C.M.: Fully coupled, multi-axial, symmetric constitutive laws for polycrystalline ferroelectric ceramics. J. Mech. Phys. Solids 50, 127-152 (2002)

22. McMeeking, R.M., Landis, C.M.: A phenomenological multiaxial constitutive law for switching in polycrystalline ferroelectric ceramics. Int. J. Eng. Sci. 40, 1553-1577 (2002)

23. Landis, C.M.: Non-linear constitutive modeling of ferroelectrics. Curr. Opin. Solid State Mater. Sci. 8, 59-69 (2004)

24. Miller, R.C., Weinreich, G.: Mechanism for the sidewise motion of $180^{\circ}$ domain walls in barium titanate. Phys. Rev. 117, 1460 1466 (1960)

25. Hayashi, M.: Kinetics of domain wall motion in ferroelectric switching. I. General Formulation. J. Phys. Soc. Jpn. 33, 616628 (1972)

26. Zhong, W.L.: Physics of Ferroelectrics (in Chinese). Science Press, Beijing (1996)

27. Zhang, Y.: On the spontaneous configuration of ferroelectricferroelastic materials (in Chinese). Chin. J. Theor. Appl. Mech. 32(2), 213-222(2000)

28. Hannes, K., Herbert, B.: On the local and average energy release in polarization switching phenomena. J. Mech. Phys. Solids 49, 953 978 (2001)

29. Li, F.X., Fang, D.N.: Effect of lateral pressure on the non-linear behaviour of PZT ceramics. Sci. China Ser. E 35(11), 1193 1201 (2005)

30. Lynch, C.S.: The effect of uniaxial stress on the electromechanical response of 8/65/35 PLZT. Acta Mater. 44, 41374148 (1996)

31. Fang, D.N., Mao, G.Z., Li, F.X. et al. : Experimental study on electro-magneto-mechanical coupling behavior of smart materials. J. Mech. Strength 27(2), 217-226 (2005)

32. Wang, Q., Chen, C.Q., Shen, Y.P.: An experimental investigation into the complex electromechanical behavior of PZT53. Chin J. Theor. Appl. Mech. 37(4), 413-420 (2005) 